\title{
Editorial
}

\section{What is the "Cutting Edge" of Social Work?}

\author{
James G. Daley
}

\begin{abstract}
$\mathbf{S}$ cial Work is a proud, complex profession with a broad, sweeping mandate. very time some theorist or academic scholar or street-wise advocate asserts the "mission" of Social Work, a flood of alternative interpretations pour in. As scholars are busy building "evidence-based practice models," post-modern cynics naysay the premise of science. Advocates of best-practice protocols butt heads with zeal ots of the "Social Work is an art" camp. This conflict was raging in 1986 when I earned my doctorate. Today's swirl of arguments has a lot in common with thefiery debates of my doctoral class. Capturing the essence of Social Work seems clearly confirmed by each person's passion for a cause but seems rarely affirmed by a majority.
\end{abstract}

There have been clear advances since 1986. There are a growing number of research centers at schools of social work. Hopefully, there will soon be an NIMH Social Work Research Center. There have been myriad studies on the effectiveness of Social Work practice. There have been dozens of textbooks on practice, policy, and advocacy. Various authors have even asserted their own models of Social Work. But have we made progress? The same arguments are being made that were made in 1986 (and probably in 1916). We are Science; we are Art. We are a professional service; we are "save-the-disenfranchised" missionaries. Work within the system; fight the system as outsiders.

As teachers, we face a fascinating challenge. We teach the professional skills, while flaming the inner fires of advocacy. We teach strategies for clinical intervention, while cautioning against blind acceptance of the diagnosis. We teach how to use the DSM, while discussing the stigma of labeling and the importance of context. We prepare the next generation of social workers to be professionally prepared for both clinical and advocacy roles.

The above beef stew of issues sets the stage for my question: what is the "cutting edge" of Social Work? As editor, I strive for articles that stimulate the reader and push the edge of our knowledge. But what cutting edge should I look for?

One argument would be that the best article is the one that systematically builds on existing research literature and adds a small piece to the slowly emerging theory or model. Empirical articles with large sample sizes, multi-site locations, and precise measures that are directly linked to the statistically confirmed model would step to the front of the line. A series of articles that repeatedly confirm the model would add credence that the model is the cutting edge.

Another argument would bethat the best article is the bold, new direction previously untapped or perhaps even conceived. Such an article puts old ideas or strategies together in a unique way. The article would shake our paradigms to the core. No data would be necessary, just clear, thoughtful, provocative ideas.

As you look at this issue, have the authors pushed to the cutting edge? Besthorn and Saleeby's fascinating review of and advocacy for biophilia is certainly thought provok- 
ing. Collins' in-depth discussion of the issues that child victims of violence face is invaluable in bringing the reader up-to-speed on the topic. Both Early, Vonk, and Kondrat's multi-cultural practice articleand Dalton and Wright's empirical exploration of redundancy in a school of social work give the reader some data-based ideas that can improve how we teach students. But are they cutting edge articles?

I would advocate that the articles are cutting edge. My reasoning is simple. Do they provoke new ideas? Do their findings encourage us to re-evaluate how we do business? Do they add to our understanding of an issue, perhaps even prompt us to read more on that issue? Are they eloquent arguments from authors who are passionate about their topic? I submit that all of the articles in this issue (and previous issues) meet the criteria in these questions. They make us think. They give us a chance to advance our skills or viewpoints.

So what is the "cutting edge" of Social Work? We bump up against it every day. The cutting edge is the new and stimulating. It might be an evidence-based skill. It might be a bold new idea. It might be new data that suggests we should change how we do business. It could be marching together to a state capitol to advocate for change. The cutting edge is not defined by camp leaders or by excluding people who do not think the "right" way. For a complex profession, the cutting edge is multi-faceted and embracing. For Social Work, the profession that prides itself with being inclusive, the cutting edge is a place that welcomes scienceand art, advocateand clinician, the dataobsessed, and the dreamer. As editor, I hope the cutting edge is Advances in Social Work.

So, to the reader I give this simple invitation. Sit back in a comfortable chair and begin to read this issue. I promise you will be tasting the cutting edge. 